Trauma Berufskrankh 2004 - 6 [Suppl 4] : S417-S423 DOI 10.1007/s10039-004-0914-5

Online publiziert: 15. Juli 2004

(c) Springer-Verlag 2004
F. Molsberger ${ }^{1} \cdot$ J.Winkler ${ }^{2} \cdot$ A. Molsberger ${ }^{3}$

${ }^{1}$ Berlin

2Lüneburg

${ }^{3}$ Düsseldorf

\title{
Akupunktur in der Unfallchirurgie
}

\section{Außenseitertherapie oder etablierte Ergänzung?}

\section{Heutige Bedeutung der Akupunktur}

Die Akupunktur zählt heute in Deutschland im ambulanten Bereich zu den beliebten und weit verbreiteten Therapieformen. 90\% aller Schmerzambulanzen und mehr als 50\% der Orthopäden wenden die Akupunktur in Deutschland an.

In der Unfallchirurgie spielt die Akupunktur bisher eine untergeordnete Rolle. In den Rettungsstellen fehlen in der Regel geeignete Räumlichkeiten, ärztliche Ressourcen und die notwendige Ausbildung für die Integration der Akupunktur in die tägliche Patientenversorgung. So wird die Möglichkeit einer sofort analgetischen Akupunktur z. B. bei einem Thoraxprelltrauma in den Kliniken bisher kaum eingesetzt.

Nach eigenen Recherchen wenden von 40 zufällig ausgewählten unfallchirurgischen Kliniken zwei Abteilungen die Akupunktur an, eine davon in der unterstützenden postoperativen Schmerztherapie im Rahmen einer Schmerzambulanz.

Ein großer Teil der ambulanten Akupunkturbehandlungen entfiel 2002 und 2003 auf die Modellvorhaben der Krankenkassen. An den Modellvorhaben nehmen über 12.00o Ärzte teil. Chronische Rückenschmerzen, Kopfschmerzen und osteoarthritische Beschwerden fallen unter die Indikationen, für die der Bundesausschuss der Ärzte und Krankenkassen die Genehmigung für die Aufnahme in die Modellvorhaben erteilt hat. Die Kosten der GKV betrugen allein für die Modellvorhaben 2002 ca. 200-270 Mill. €jährlich (1,0-1,4\% des Budgets für Medikamente und Hilfsmittel [19]). Zusätzlich erbringen die etwa 40.000 Ärzte, die Akupunktur anbieten (30\% aller niedergelassenen Kollegen), Leistungen für privat Versicherte oder selbst zahlende Patienten. Diese Leistungen werden vornehmlich bei der ambulanten Versorgung chronisch kranker Patienten erbracht und sind als Schmerztherapie häufig Bestandteil des Leistungskataloges der konkurrierenden Privatkassen. Zunehmend erstatten Kostenträger die Akupunktur auch bei Nichtschmerzerkrankungen, z. B. Allergie oder Schwangerschaftserbrechen.

In letzter Zeit fragen Ärzte und Patienten den Autor vermehrt nach Therapien für die ambulante postoperative Nachsorge, d. h. Schmerztherapie, Therapie funktioneller Einschränkungen und allgemeiner Befindlichkeitsstörungen in der Rekonvaleszenz.

\section{Geschichtlicher Hintergrund}

\section{Akupunktur - eine energetische Medizin mit langer Tradition}

Die Ärzteschaft entwickelte die Akupunktur aus der chinesischen Medizin vor ca.
2000 Jahren in einem - bis heute währenden - unkontrollierten Feldversuch unter Einbeziehung konfuzianischer Vorstellungen [21]. Sie ist damit eine empirisch und historisch gut begründete Therapiemethode. Die kontinuierliche Weiterentwicklung der Akupunktur über die Jahrhunderte in China spricht für einen therapeutischen Nutzen und vor allem gegen ausgeprägte unerwünschte Wirkungen. Das „Argument der Evolution“ begründet das in den letzten Jahrzehnten über alle kulturelle Schranken wachsende Interesse an der Akupunktur. Große wissenschaftliche Untersuchungen zur Akupunktur, wie z. B. die Gerac („German acupuncture trial") und die ART-Studie, sind in Deutschland ins Leben gerufen worden. Der Versuch, die Akupunktur in das westliche Denkmuster zu integrieren, und die Notwendigkeit kontrollierter Modellversuche werden aber auch kontrovers diskutiert [22].

\section{Traditionelle Wirkungsweise}

\section{Yin, Yang und Q}

Die traditionelle chinesische Medizin (TCM) beruht auf den Konzepten von Yin, Yang, Qi (gesprochen Tschi) und Leitbahnen, sog. Meridianen [5, 21].

In der traditionellen bildhaften Beschreibung von Yin und Yang ist 


Yang Yin

- Außen

- lateral

- Spitz

- ausstrahlend

- Rot

- Hitze

- Bewegung

- Anspannung

- Hyper

- Fülle

- Struktur
- Innen

- medial

- Dumpf,

- zusammenziehend

- Livide

- Kälte

- Ruhe

- Entspannung

- Hypo

- Leere

- Funktion
Abb. $1<$ Yin und Yang in der Medizin

\section{Der Blasenmeridian \\ Zweiter Umlauf (He - Dü - Bl - Ni) Beginn: medialer Augenwinkel (BI 1) \\ Verlauf: Stirn, Kopf, dorsal an Nacken, Rücken und Bein zur 5 . Zehe \\ Ende: 5. Zehe, Nagelfalz lateral (BI 67) \\ Innerer Verlauf: im Lumbalbereich zur Niere und Blase}

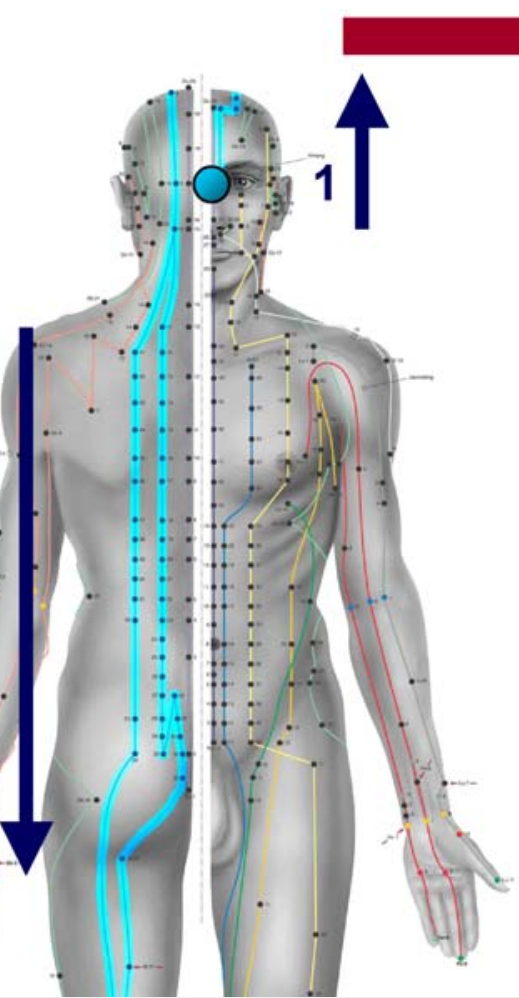

- Yang die „von der Sonne beschienene Seite des Berges" und

- Yin die „Schattenseite des Berges“.

Die chinesische Medizin beschreibt für die Polaritäten Yin und Yang eine Vielzahl möglicher Interaktionen (• Abb. 1). Da-bei ist die Bewertung immer vom Standpunkt und Blickwinkel des Betrachters abhängig. Beispielsweise kann handwarmes Wasser als Yang im Vergleich zu Eis (Yin) oder als Yin gegenüber Wasserdampf (stärkeres Yang) gesehen werden.
Die Lebensenergie Qi umfasst Yin- und Yang-Aspekte und zirkuliert in 14 Meridianen durch den Körper.

Gesundheit be- und entsteht aus Sicht der chinesischen Medizin

- durch ein Gleichgewicht von Yin und Yang,

- durch einen harmonischen Qi-Fluss und

- durch eine zirkadian ausgewogene Rhythmik.
Abb. $2<$ Der Blasenmeridian. Mit dem Blasenmeridian werden u. a. akute und chronische Schmerzsyndrome der ganzen Wirbelsäule behandelt 
Fraktur und Osteoporose aus Sicht der chinesischen Medizin

$\mathrm{Zu}$ einer Fraktur kann es aus Sicht der chinesischen Medizin durch zwei Interaktionen von Yin und Yang kommen:

Fraktur durch Trauma. Das Trauma als zu starkes Yang führt zu einer Destruktion der gesunden Struktur Yin (Knochen). Indem wir die Fraktur ruhig stellen, stärken wir das Yin und halten Yang (Bewegung) von dem Knochen fern. Nach der YinKonsolidierung wird der Gips oder Fixateur entfernt. Jetzt ist die Extremität in ihrer Funktion Yang eingeschränkt. Die erforderliche Krankengymnastik gleicht diesen Yang-Mangel aus. Die zunehmende angepasste Yang-Belastung fördert wiederum die weitere Yin-Festigung.

Fraktur bei Osteoporose. Die chinesische Medizin wertet Osteoporose als Yin-Mangel. Das schwache Yin hält einer Yang-Alltagsbelastung nicht stand. Die schulmedizinische medikamentöse orthomolekulare Therapie dient dem Yin-Aufbau. Maßvoll angepasstes Yang stabilisiert das Yin ebenfalls. In der chinesischen Medizin werden bei Yin-Mangel-Erkrankungen häufig zusätzlich Kräuter eingesetzt [4].

Pragmatisches schulmedizinisches Denken und traditionelle Yin- und Yang-Dialektik führen in diesen Beispielen gleichermaßen zu sinnfälligen Schlussfolgerungen (•Abb. 3).

Die Yin- und Yang-Dialektik der chinesischen Medizin hat in der deutschen Naturheilkunde eine prominente Entsprechung: Pfarrer Kneipp als einer der Wegbereiter der physikalischen Therapie beschrieb mit der Ordnungstherapie das Wechselspiel von Reiz und Ruhe als eine der wichtigsten Vorraussetzungen für das Gedeihen des menschlichen Organismus [2].

\section{Wissenschaftlicher Hintergrund}

\section{Grundlagenwissenschaftliche Erkenntnisse}

Nadeleinstiche stimulieren die lokalen peripheren Rezeptoren. Afferente, dünne myelinisierte Nervenfasern leiten den Reiz,

Trauma Berufskrankh 2004 - 6 [Suppl 4] : S417-S423

DOl 10.1007/s10039-004-0914-5

(C) Springer-Verlag 2004

F. Molsberger $\cdot$ J. Winkler $\cdot$ A. Molsberger

\section{Akupunktur in der Unfallchirurgie. Außenseitertherapie oder etablierte Ergänzung?}

\section{Zusammenfassung}

Die Akupunktur zählt in Deutschland im ambulanten Bereich zu den beliebten und weit verbreiteten Formen der Schmerztherapie. Sie spielt bisher in der Unfallchirurgie eine untergeordnete Rolle und könnte vermehrt in der Rettungsstelle als additives Therapieverfahren stationär präoperativ und postoperativ eingesetzt werden. Hier gilt es, spezielle deblockierende und detonisierende Techniken zur Schmerztherapie mit rascher Wirksamkeit einzusetzen, regenerative
Mechanismen zu triggern und die günstige Wirkung auf die Psyche zu nutzen. Es müssen Mindestanforderungen an die Ausbildung und an einen einheitlichen Therapiestandard gestellt werden. Weitere Studien zur Akupunktur in der Unfallchirurgie sind notwendig.

\section{Schlüsselwörter}

Schmerztherapie · Akupunktur · Detonisierung Deblockierung

\section{Acupuncture in trauma surgery. Maverick treatment or established adjuvant?}

\section{Abstract}

Acupuncture ranks among the most popular and widespread treatment forms employed in Germany on an outpatient basis for pain therapy. Up to now it has played a subordinate role in trauma surgery and could be utilized more in the emergency center as an additional inhospital treatment procedure for preoperative and postoperative care. Effective use entails applying special deblocking and detoning techniques for rapidly acting pain therapy, triggering regenerative mechanisms, and taking advantage of the beneficial effect on the psyche. Minimum requirements must be imposed for training and a uniform treatment standard. Further studies on acupuncture in trauma surgery are needed.

\section{Keywords}

Pain therapy · Acupuncture · Detoning .

Deblocking 


\section{Yin und Yang Fraktur und Osteoporose}

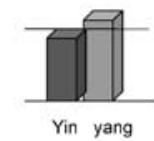

Krankheitsverlauf bei einer Fraktur durch adäquates Trauma

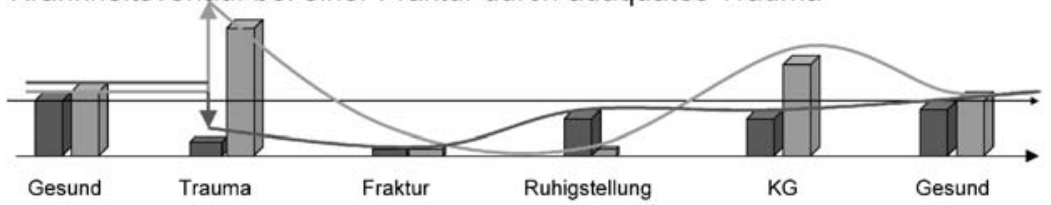

Krankheitsverlauf bei Osteoporose

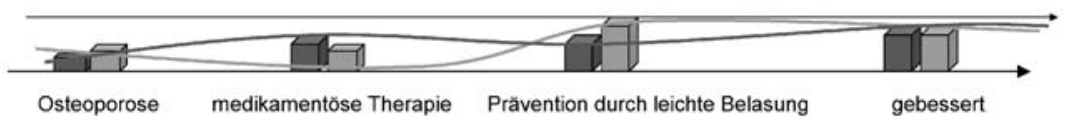

Abb. $3 \Delta$ Yin und Yang im Verlauf bei Osteoporose und Fraktur

der durch den Nadelstich an Akupunkturpunkten über periphere Rezeptoren gesetzt wurde, an das Hinterhornneuron weiter. Im Rückenmark wird die Erregung auf segmentaler Ebene auf ein zweites Neuron umgeschaltet und erreicht über den Tractus spinothalamicus das Zentralnervensystem. Segmental setzt die Akupunktur mindestens die Neurotransmitter Enkephalin und Dynorphin und im Bereich des ZNS mindestens $\beta$-Endorphin und Serotonine frei. Diese neurophysiologischen Mechanismen sind in der Literatur sehr gut belegt. Die Modulation der Schmerzwahrnehmung erklärt vor allem die kurze analgetische Wirkung der Akupunktur [18].

Eine neuere Hypothese postuliert die Stimulation spezifischer Wachstumsfaktoren durch die Akupunktur. Hierzu gehören die transformierenden Wachstumsfaktoren $\beta_{1}$ und $\beta_{2}$ (TGF- $\beta_{1}$, TGF- $\beta_{2}$ ), die insulinähnlichen Wachstumsfaktoren IGF-1 und IGF-2, der „,bone-derived growth factor" (BDGF), der Fibroblasten- (FGF) und der Nervenwachstumsfaktor (NGF). Diese immunologischen Mechanismen könnten erstmalig die bei chronischen Erkrankungen des Bewegungsapparates zu beobachtende langfristige regenerative Wirkung der Akupunktur erklären [11].

\section{Ergebnisse klinischer Forschung}

Kontrollierte und nichtkontrollierte Studien zur Akupunktur wurden in den letzten 20 Jahren vielfach publiziert. Die meisten Arbeiten weisen bezüglich der metho- dischen, biometrischen oder handwerklichen Qualität Mängel auf.

Genauso selten wie im Bereich der konventionellen Medizin sind prospektive, kontrollierte, randomisierte, wenn möglich verblindete Studien nach den Prinzipien der "good clinical practice" (GCP) zu finden - klinische Studien also, die strengen wissenschaftlichen Ansprüchen der evidenzbasierten Medizin im Bereich der Akupunktur genügen [20]. Nach Gerlach sind aber auch in der westlichen Medizin nur knapp $4 \%$ aller Therapieverfahren durch Wirksamkeitsnachweise belegt [6]. Vor diesem Hintergrund ist die klinisch-wissenschaftliche Situation in der Akupunktur etwa derjenigen der westlichen Medizin vergleichbar.

Seit den 9oer-Jahren des vorigen Jahrhunderts sollen öffentliche Förderprogramme („unkonventionelle medizinische Richtungen “,Forschungsförderprogramm des BMBF) den Mangel an guten klinischen Studien beheben. Publikationen geförderter Studien verbesserten in den letzten Jahren die Nachweislage zur Akupunktur. Dies gilt für die Indikationen Halswirbelsäulensyndrom sowie chronischer Kreuz- und Schulterschmerz [7, 9, 13].

Das National Institute of Health (NIH, USA) konstatierte 1997 in seinem Consensus Development Statement das Fehlen gesicherter wissenschaftlicher Nachweise zur Akupunktur bei Erkrankungen des Bewegungsapparates. Studien mit gutem Design, die unabhängige Arbeitsgruppen durchgeführt hatten, wurden gefordert. Das NIH kam zur Auffassung, dass gute Studien im Bereich des Bewegungsapparates nur für eine analgetische Akupunkturwirkung bei Tennisarm und Fibromyalgie existierten [17].

Der HTA-Bericht des Bundesausschusses der Ärzte und Krankenkassen im Januar 2001 kam in seiner Bewertung der Akupunktur zu dem Ergebnis, dass sich Hinweise für eine mögliche Wirksamkeit, die eine weitere klinische Erforschung begründeten, für Spannungskopfschmerz, Migräne, Arthrose und Kreuzschmerz fänden [1].

Aufgrund des Ergebnisses dieses Berichtes verpflichtete der Bundesausschuss der Ärzte und Krankenkassen im Oktober 2000 die GKV, die Akupunkturbehandlung nur noch im Rahmen von Modellprojekten zu bezuschussen. Die Wirksamkeit der Akupunktur bei chronischem Kreuz-, Gonarthrose- und Spannungskopfschmerz sowie bei Migräne solle so wissenschaftlich erforscht werden. Daraus resultieren die eingangs erwähnten Modellvorhaben.

\section{Akupunktur in der Unfallchirurgie}

\section{Wenige Akupunkturstudien zu unfallchirurgischen Indikationen}

Bei der Behandlung des komplexen regionalen Schmerzsyndroms (CRPS) konnte Korpan bei 14 Patienten keinen Unterschied zwischen der Verum- (der TCM entsprechenden Akupunktur) und der Sham-Akupunktur (Akupunktur an „falschen " Punkten, aber mit echten Nadeln) feststellen und fordert weitere Untersuchungen [8].

Bei der Behandlung des Tennisarms ist die sofort analgetische Wirkung der Meridianakupunktur nachgewiesen [12].

Für die in der Unfallchirurgie häufigen Indikationen mit speziellen sofort analgetischen und deblockierenden Techniken (One-point-Akupunktur) und für viele andere klinisch häufige Akupunkturindikationen wurden bisher noch keine kontrollierten Studien durchgeführt.

Die Frage des Wirksamkeitsnachweises wurde damit nicht gestellt und kann auch nicht beantwortet werden. Keinesfalls darf dies jedoch mit einer erwiesenen Unwirksamkeit gleichgesetzt werden. 


\section{Integration in die unfallchirurgische Praxis}

Die Akupunktur wird auch in der Unfallchirurgie zukünftig eine wichtigere Rolle spielen. Die Wirksamkeit der Akupunktur bei unfallchirurgischen Indikationen muss durch weitere Studien untersucht werden.

\section{Ambulanz}

Die Akupunktur eignet sich zunächst prinzipiell für alle Patienten, die eine unfallchirurgische Einrichtung ohne operationsbedürftiges Trauma oder mit einer geringgradigen Verletzung oder mit Schmerzen des Bewegungsapparates aufsuchen.

Gerade für die ambulante Versorgung von Sportverletzungen, akuten lokalen Schmerzen und Blockierungen gibt es vielfach erprobte Therapiekonzepte.

Hier ist die rasch wirksame, schmerzlindernde, deblockierende und detonisierende One-point-Akupunktur als sofort analgetische Stimulationstechnik zu nennen. Es gilt, die Vorteile der Akupunktur mit denen der üblichen diagnostischen und therapeutischen unfallchirurgischen Maßnahmen zu kombinieren und die $\mathrm{Pa}$ tienten nach der Akutversorgung rasch in die gebiets- oder hausärztliche Behandlung zu entlassen.

\section{Klinisches Beispiel}

So wird beispielsweise das akute Thoraxprelltrauma - sei es bei einem Eishockeyspieler, sei es nach einem Fahrradunfall mit dem Punkt San Jiao 8 am Unterarm behandelt (• Abb. 4). Der oft innerhalb von Sekunden eintretende Therapieerfolg kann bereits anhaltend sein. Hintergrund für diese für Therapeuten und Patienten oft verblüffenden Erfolge ist aus Sicht der chinesischen Medizin die Korrespondenz longitudinaler Körperachsen über das Meridiansystem [16].

\section{Stationär}

Bei der Behandlung von Verletzten, die einer stationären operativen oder konservativen Versorgung bedürfen, kann die Akupunktur vermehrt als supportive Schmerztherapie eingesetzt werden. Der von Akupunkturärzten immer wieder beobachtete günstige Einfluss auf die Psyche, z. B. mit dem Punkt Du 20 auf der

\section{San Jiao 6 und 8 Beispiel für Indikationen}

SJ 6 Verzweigter Graben Zhigou

Lokalisation: 3 Cun (4 Querfinger) proxima der dorsalen Handgelenksquerfalte zwischen Radius und UIna

Indikation: u.a.: „One-point" bei akuter Nackensteifigkeit

Punkt ca. 2 Min. stimulieren

SJ 8 Luo-Verbindung der drei Yang Sanyangluo

Lokalisation: 4 Cun (5,5 Querfinger) proximal der dorsalen

Handgelenksquerfalte $z w$. Radius und Ulna Indikation:One-Point" für Rippenprellung (-fraktur)

Punkt ca. 2 Min. stimulieren

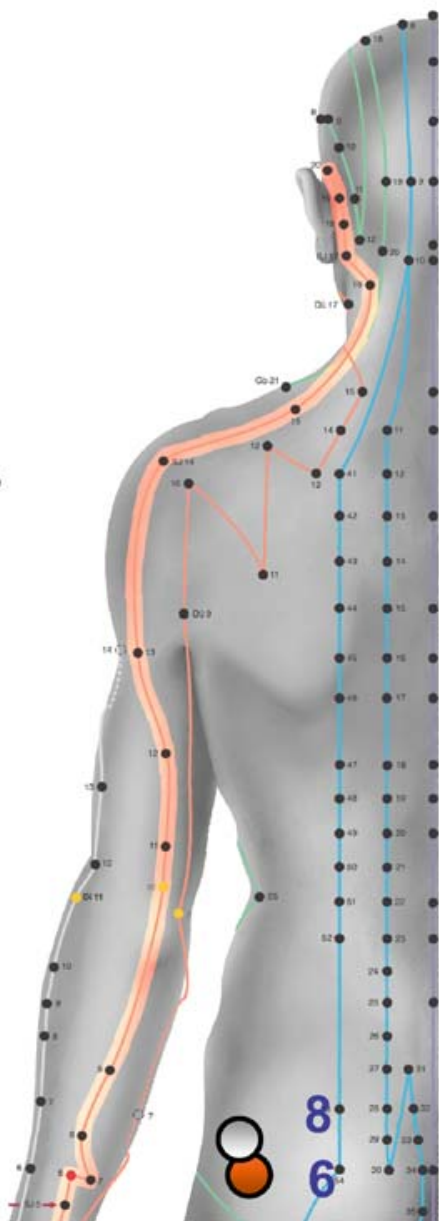

Abb. $4 \Delta$ San Jiao 6 und 8 - Beispiel für Indikationen. Der Punkt wird bei einem Thoraxprelltrauma ipsilateral streckseitig ca. eine Handbreite vom Handgelenk etwa $1 \mathrm{~cm}$ tief genadelt und die Nadel dabei durch Rotation sowie Heben und Senken für $\mathbf{2}$ min stimuliert. Während der Stimulation lässt der Therapeut den Patienten vorsichtig tiefer durchatmen und das Qi der betroffenen Region mit der Hand verreiben

Spitze des Kopfes, kann vermehrt psychovegetativ regulierend genutzt werden. Darüber hinaus lässt sich mit der Akupunktur Wartezeit sinnvoll überbrücken und nutzen, der Patient erlebt sie als Zeit ärztlicher Zuwendung und Therapie.

\section{Unfallchirurgische Indikationen im Überblick}

\section{Indikationen in der Rettungsstelle/ Ambulanz/Sportmedizin}

\footnotetext{
- Mobilisation bei Gelenkblockierungen,

- Thoraxprelltrauma,

- Rippenfraktur,

- Mobilisation nach Schultertrauma,

- Reposition nach Schulterluxation,

- HWS-Schleudertrauma,
}

- Lumbago und Lumboischialgie und

- Schmerztherapie

Indikationen in der

präoperativen Phase

- Schmerztherapie und Mobilisation (Indikationen wie in der Ambulanz),

- psychovegetativ ausgleichende Wirkung sowie

- Therapieangebot und (ärztliche) Zuwendung in der Wartezeit

\section{Indikationen in der} postoperativen Phase

- Schmerztherapie und Mobilisation (Indikationen wie in der Ambulanz), - rascheres Abschwellen traumatisierten Gewebes, 
- Muskelrelaxation und Mobilisation,

- enge Kooperation mit Krankengymnastik,

- postoperative Übelkeit,

- atonische Blase mit Harnverhalt,

- Stärkung der „Körpermitte“ und Gewinnen von Vertrauen in den eigenen Körper zur rascheren Rehabilitation,

- gezieltes Triggern regenerativer Mechanismen,

- das Konzept von Yin und Yang zur Optimierung des Therapieplanes,

- Yamamoto-Schädelakupunktur (YNSA) bei Zustand nach SchädelHirn-Trauma und

- chinesische Schädelakupunktur bei Zustand nach Schädel-Hirn-Trauma

\section{Anzahl der Akupunktursitzungen}

\section{Akute Erkrankung: 1-6 Sitzungen}

Bei akuten Erkrankungen lässt sich der Schmerz in einer einzigen Sitzung von wenigen Minuten mit der oben beschriebenen One-point-Akupunktur oft um mehr als 50\% reduzieren. Geeignete Messinstrumente sind die Angaben des Patienten gemäß der visuellen Analogskala (VAS) sowie der körperliche Untersuchungsbefund. Nach der Akutbehandlung in der Rettungsstelle kann die Therapie von den ambulant weiterbehandelnden Ärzten fortgesetzt werden. Häufig reichen insgesamt 1-6 Therapiesitzungen bei maximal einer Sitzung pro Tag aus.

\section{Chronische Erkrankung: 15 Sitzungen}

Chronische Schmerzerkrankungen erfordern in der Regel mindestens 15 Sitzungen, wobei nach internationalem Expertenkonsens eine erste Besserung zwischen der 6. und 10. Sitzung zu erwarten ist [14]. Negative Therapieergebnisse - wie auch unklare Studienergebnisse in der Vergangenheit - sind häufig durch eine zu geringe Anwendungsanzahl der Akupunkturen bedingt. Dies belegt auch eine retrospektive Studie an 163 Patienten mit chronischen Schmerzerkrankungen (mittlere Erkrankungsdauer 6,3 Jahre), in der gezeigt wurde, dass bei Patienten, die sich selbst als erfolgreich behandelt bezeichneten, im Mittel 17,8 Therapiesitzungen erforderlich gewesen und erste Zeichen der Besserung im Mittel nach 9,8 Sitzungen aufgetreten waren [10].

\section{Implementierung in die Klinik}

Voraussetzung für eine Implementierung in den Klinikalltag ist eine entsprechende Schulung der Ärzte. Als eine Maßnahme zur Sicherung der Qualität hat die BÄK 2004 die Einführung der facharztbezogenen Zusatzweiterbildung „Akupunktur“ mit 200 Ausbildungsstunden bundesweit beschlossen [3]. Im Curriculum sind das Erlernen des Meridiansystems und grundlegende Techniken der Schmerzbehandlung vorgesehen. Damit ist die Ausbildung für unfallchirurgische Indikationen ausreichend.

Die Methode ist bei entsprechender Ausbildung fast überall einsetzbar. Zur Durchführung werden lediglich Akupunkturnadeln benötigt.

\section{Einsatzmöglichkeiten der sofort analgetischen Stimulationstechnik}

- Rettungsstelle,

- Operationssaal,

- Station und

- ambulante Nachsorge

Sicherstellung der Akupunkturqualität Im Konsens mit führenden nationalen und internationalen Experten sowie der maßgeblichen Literatur wurden für die Gerac Leitlinien zur Akupunkturtherapie entwickelt [15]. Hieraus ergeben sich auch für unfallchirurgische Indikationen folgende Anforderungen an eine qualitätsbewusste Akupunkturbehandlung (die Punkte 4-7 betreffen vor allem die stationäre und ambulante Rehabilitation):

1. Die Indikationsstellung für eine Akupunkturtherapie erfolgt immer unter Einbeziehung der fachmedizinischen Diagnostik. Die Akupunktur wird selten als Monotherapie und meist in Kombination mit anderen bewährten fachmedizinischen Therapieverfahren eingesetzt.

2. Die Ashi-Punkte (Locus-dolendiPunkte) werden genau palpiert und den betreffenden Akupunkturmeridianen zugeordnet. Die Punktauswahl erfolgt zuerst nach der Meridiantheorie.

3. Das Krankheitsbild wird nach den Kriterien der chinesischen Medizin -
Blut und Qi - sowie nach den BaGang-Kriterien - Yin und Yang, Leere und Fülle, Kälte und Hitze, innere und äußere Störung - eingeordnet.

4. Bei chronischen Erkrankungen mit pathologischen Befunden in den chinesischen Syndromen erfolgt die Nadelung zusätzlicher energetische Punkte. Ggf. wird ergänzend ein schriftlicher Ernährungsplan nach Erkenntnissen der chinesischen Medizin und Rezeptur entsprechend der chinesischen Phytotherapie erstellt.

5. Bei chronischen Erkrankungen mit Beteiligung mehrerer Körperregionen wird die chinesische Syndromdiagnostik einschließlich Zungen- und ggf. Pulsdiagnostik durchgeführt.

6. Ein Therapieplan mit Festlegung von in der Regel 1-20 Akupunkturpunkten und der voraussichtlichen Anzahl und Frequenz der Akupunktursitzungen (s. o.) wird erstellt. Neben der Punktauswahl sind die Stichtiefe sowie die Art und Intensität der Nadelstimulation - manuell, durch Wärme (Moxibustion), Schröpfköpfe oder elektrisch - zu bestimmen.

7. Weitere ergänzende Verfahren, wie die Bewegungstherapie Qi Gong und die chinesische Massagetechnik Tuina, können zusätzlich eingesetzt werden.

8. Alle therapeutischen Anwendungen werden detailliert dokumentiert insbesondere Akupunkturpunkte und Stimulationstechnik.

9. Das Therapieergebnis wird evaluiert.

\section{Fazit}

- Die Akupunktur wird sich als additives Therapieverfahren auch in der Unfallchirurgie etablieren.

- Der sofort analgetische Effekt der Akupunktur eignet sich besonders für die ambulante Versorgung nicht operationsbedürftiger Schmerzpatienten.

- Die Akupunktur als Schmerztherapie und psychisches Regulans kann in der gesamten stationären Versorgung eingesetzt werden.

- Erkrankungen des Bewegungsapparates sind das Hauptindikationsgebiet der Akupunktur. 
- Die Akupunktur hat sich zu einer klinisch etablierten, bei Arzt und Patient beliebten Therapieform entwickelt.

- Die Nachweislage zur Wirksamkeit der Akupunktur entspricht derjenigen der konventionellen Medizin. Weitere Studien zur Verbesserung der wissenschaftlichen Nachweislage sind erforderlich.

- Entscheidend für den Therapieerfolg ist die Einhaltung definierter Qualitätskriterien.

- Die Akupunktur erweitert das therapeutische Spektrum der westlichen Medizin. Sie wird zusammen mit bewährten westlichen Therapieformen eingesetzt.

\section{Korrespondierender Autor Dr. F. Molsberger}

und die Forschungsgruppe Akupunktur, Tristanstraße 42, 14476 Groß Glienicke, E-mail: F.Molsberger@facm.de

Interessenkonflikt: Keine Angaben

\section{Literatur}

1. Arbeitsausschuss „Ärztliche Behandlung" des Bundesausschusses der Ärzte und Krankenkassen (2001) Akupunktur. Zusammenfassender Bericht der Geschäftsführung des Arbeitsausschusses „Ärztliche Behandlung" des Bundesausschusses der Ärzte und Krankenkassen, Köln

2. Bachmann RM, Schleinkofer GM (1997) Natürlich gesund mit Kneipp. Thieme, Stuttgart, p 151

3. Bundesärztekammer (2003) Weiterbildungsordnung

4. Ehling D (2001) Handbuch chinesische Kräuterrezepte. Urban \& Fischer, München

5. Focks C, Hillenbrand N (2000) Leitfaden traditionelle Chinesische Medizin, Schwerpunkt Akupunktur, 2. Auflage. Fischer, Ulm

6. Gerlach FM (2001) Qualitätsförderung in Praxis und Klinik - eine Chance für die Medizin, 1. Aufl. Thieme, Stuttgart

7. Irnich D et al. (2001) Randomised trial of acupuncture compared with conventional massage and „sham" laser acupuncture for treatment of chronic neck pain. BMJ 322:1574-1578

8. Korpan M et al. (1999) Acupuncture in the treatment of posttraumatic pain syndrome. Acta Orthop Belg 65: 197-201

9. Leibing E et al.(2002) Acupuncture treatment of chronic low-back pain - a randomized, blinded, placebo-controlled trial with 9-month follow-up. Pain 96:189-196

10. Molsberger A (1992) Anzahl der Sitzungen bis zum Therapieerfolg.Vortrag Kassenärztliche Bundesvereinigung

11. Molsberger A (1994) Mit Neuroakupunktur gegen Radikulitiden. Z Allg Med 70:1-3

12. Molsberger A, Hille E (1994) The analgesic effect of acupuncture in chronic tennis elbow pain. Br J Rheumatol 33:1162-1165

13. Molsberger A et al. (2002) Does acupuncture improve the orthopedic management of chronic low back pain - a randomized, blinded, controlled trial with 3 months follow-up. Pain 99:579-587
14. Molsberger A et al. (2002) Acupuncture in the treatment of locomotive disorders - status of research and situation regarding clinical application. Schmerz 16: 121-128

15. Molsberger A et al. (2002) GERAC-Akupunktur-Studien - Modellvorhaben zur Beurteilung der Wirksamkeit. Dtsch Ärzteblatt 99:26

16. Molsberger F (2001) Bi-Syndrome, Skript zur Vollausbildung

17. NIH Consensus Conference (1998) Acupuncture. JAMA 280:1518-1524

18. Pomeranz BH (2000) Acupuncture analgesia - basic research. In: Stux G, Hammerschlag R (eds) Clinical acupuncture - scientific basis. Springer, Berlin Heidelberg New York Tokyo

19. Rieser S (2001) Neue Therapieansätze - unzulässige Zahlungen. Dtsch Ärzteblatt 98A:1224

20. Stux G (2001) Proposed standards of acupuncture treatment for clinical studies. In: Stux G, Hammerschlag $\mathrm{R}$ (eds) Clinical acupuncture - scientific basis. Springer, Berlin Heidelberg New York Tokyo

21. Unschuld PU (1997) Chinesische Medizin. Beck, München

22. Unschuld PU (2004) Akupunktur als Alternative Chance vertan? FATCM Newsletter 1:4-9 